\title{
La interactividad en los museos de ciencias, pivote entre expectativas y hechos empíricos: el caso del Centro Interactivo de Ciencia y Tecnología Abremate (Argentina)
}

\author{
Interactivity in science museums, the link between expectations \\ and empirical facts: the case of Centro Interactivo de Ciencia y \\ Tecnología Abremate (Argentina)
}

Luisa Massarani $^{1}$
https://orcid.org/0000-0002-5710-7242
María Eugenia Fazio ${ }^{2}$
https://orcid.org/0000-0003-3374-7875
Jessica Norberto Rocha ${ }^{3}$
https://orcid.org/0000-0002-9754-3874
Analía Dávila $^{4}$
https://orcid.org/0000-0002-7514-9972
Susana Espinosa ${ }^{4}$
https://orcid.org/0000-0002-0843-6971
Fabián Alejandro Bognanni ${ }^{4}$
https://orcid.org/0000-0002-8455-4195

Resumo: Este artículo reflexiona sobre la interactividad y la experiencia de los adolescentes en museos de ciencias, a través de un estudio exploratorio y cualitativo realizado en el Centro Interactivo de Ciencia y Tecnología Abremate (Argentina). El corpus recoge el registro audiovisual de las visitas realizadas por cinco grupos de estudiantes de 14 a 17 años de escuelas públicas del conurbano bonaerense. Los datos recolectados se codificaron con una herramienta orientada a comprender procesos de aprendizaje en museos de ciencias de América Latina. El análisis indica que la interactividad está presente, principalmente, en situaciones en las que los jóvenes interactúan en simultáneo con la exhibición y entre ellos, mientras conversan sobre la manipulación de los artefactos. También se observa que hay condiciones iniciales para promover el aprendizaje de actividades que enseñen a pensar y ejercitar emociones sobre la inteligibilidad del mundo.

Palabras-clave: Museo de ciencias. Educación científica. Interactividad. Actividades educativas no-escolares.

\begin{abstract}
This article reflects on the interactivity and experience of adolescents in science museums, through an exploratory and qualitative study carried out in the Interactive Center of Science and Technology Abremate (Argentina). The corpus includes the audiovisual record of the visits made by five groups of students between 14 and 17 years old from public schools in the Buenos Aires suburbs. The collected data were coded with a tool oriented towards the understanding of learning processes in science museums in Latin America. The analysis indicates that interactivity is present, especially, in situations in which young people interact simultaneously with the exhibition and with each other, while they talk about the manipulation of the artifacts. It is also observed that there are initial conditions to promote the learning of activities that teach thinking and to exercise emotions about the intelligibility of the world.
\end{abstract}

Keywords: Science museum. Science education. Interactivity. Non-school educational activities.

${ }^{1}$ Fundação Oswaldo Cruz, Programa de Pós-graduação em Divulgação da Ciência, Tecnologia e Saúde, Rio de Janeiro, RJ, Brasil. E-mail: luisa.massarani@fiocruz.br

${ }^{2}$ Universidad Nacional de Quilmes (UNQ), Departamento de Ciencias Sociales, Buenos Aires, Argentina.

${ }^{3}$ Fundação Cecierj, Programa de Pós-graduação em Divulgação da Ciência, Tecnologia e Saúde e Instituto Nacional de Comunicação Pública da Ciência e Tecnologia, Rio de Janeiro, RJ, Brasil.

${ }^{4}$ Universidad Nacional de Lanús, Centro Interactivo de Ciencia y Tecnología Abremate, Buenos Aires, Argentina. 


\section{Introducción}

La educación científica para el público joven no se da únicamente en el espacio formal de la escuela. Esta puede suceder en diversos espacios y momentos a través de la educación no formal y es aquella que ocurre de manera espontánea o semiestructurada en centros y museos de ciencias, jardines botánicos, zoológicos, acuarios, planetarios, eventos de divulgación de la ciencia, ferias de ciencias, centros culturales, teatros y cines, entre otros (MARANDINO, 2017). Los museos y centros de ciencias son fuentes importantes de aprendizaje y contribuyen a promover la cultura científica de la sociedad. De acuerdo con Cazelli y Coimbra (2008, p. 3, traducción nuestra), "la visita a museos tal vez sea la experiencia multimedia más completa. La combinación de arquitectura, textos, objetos, figuras, sonidos, música y ordenadores están diseñados para producir en el visitante una impresión extraordinaria." En este contexto, el aprendizaje que puede ocurrir en la educación no formal tiene como características el énfasis en la interacción, lo lúdico y el placer obtenido en la propia actividad, promoviendo el aprendizaje.

Las propuestas interactivas se volvieron predominantes en los museos de ciencias desde los años 1960. Una de las pioneras e inspiradoras fue la del centro de ciencias Exploratorium (Estados Unidos), que tenía como objetivo comunicar el entusiasmo por la ciencia y hacer que el visitante experimente por sí mismo el proceso científico. Su marca de origen fue la interactividad deliberadamente orientada a aprender haciendo, caracterizada por la utilización de aparatos construidos para explorar fenómenos científicos por medio del manejo, hands-on, y por la idea de que el espacio funcione como un laboratorio. Se destacó por la innovación y aplicó recursos que permitían al visitante operar botones y girar manivelas, creando estímulos para los recursos sensoriales humanos (CAZELLI; MARANDINO; STUDART, 2003).

La penetración de la interactividad también encontró terreno fértil en las necesidades institucionales por superar la "seriedad, sobriedad, aburrimiento, exceso de información y necesidad de mediación a través de guías" (OLIVEIRA et al., 2014, p. 2, traducción nuestra). Además, se presentó como una opción novedosa, estimulante, rica en evidencias, multisensorial y divertida frente a la educación formal. Y también como una estrategia para favorecer la toma de decisiones personales sin restricciones curriculares (ALLEN, 2004).

En América Latina, el aggiornamiento de los museos en este sentido demoró algunas décadas más a pesar, incluso, de la presencia y contundencia que comenzaban a tener las corrientes vanguardistas hacia mediados del siglo y, principalmente, en los años 1960. La coyuntura política y económica de la región no fue neutral en la ralentización de este proceso (CASTILLA, 2017). La interactividad recién se incorpora al diseño de los museos latinoamericanos entre los años 1970 y 1980, y continuó en los años 1990 y 2000 cuando más países de la región se sumaron a estas propuestas (MASSARANI et al., 2015). En Argentina, en particular, el Museo Participativo de Ciencias, creado en el 1988 en Buenos Aires, de carácter privado, fue el que inauguró las propuestas interactivas (PEDERSOLI, 2003). Y el Centro Interactivo de Ciencia y Tecnología (CICyT) Abremate, tomado como caso de estudio en el presente trabajo, inaugurado en el año 2001 y perteneciente a la Universidad Nacional de Lanús (Provincia de Buenos Aires), fue el primer espacio interactivo universitario y gratuito a nivel nacional.

La incorporación de la interactividad fue, además, parte de un proceso más amplio marcado por el inicio de la profesionalización y la incorporación en la gestión institucional de los estudios de públicos. Tal como señala Asensio (2017, p. 13-14), "como consecuencia de 
todo ello las prácticas comienzan a cambiar. Cambian las maneras de planificar y se empieza a notar en las exposiciones, con la irrupción de más recursos comunicativos e interactivos [...]”. Los visitantes pasan a tener un carácter activo y participativo, "por lo que es imprescindible generar los espacios de interacción pertinentes” (ASENSIO, 2017, p. 13-14). En la actualidad, además de representar un concepto y una práctica totalmente incorporados y hegemónicos, la interactividad también enfrenta nuevos debates y desafíos. Uno de ellos se vincula con las críticas a las confusiones - por parte de los propios museos, los públicos y el campo académico dedicado a comprender los procesos en los museos de ciencias (ROWE; O'BRIEN, 2016) entre interactividad y sofisticación tecnológica (OLIVEIRA et al., 2014).

Otro debate hurga directamente en qué es y qué se entiende por interactividad, y desmenuza la diversidad de calidades y niveles de profundidad que puede alcanzar. Es así que varios autores distinguen grados de manipulación y discriminan, por ejemplo, diferentes niveles de actividades interactivas. En los niveles más bajos de interacción están aquellas actividades que se asocian a intervenciones simples (como tocar un botón o mover una manivela) y a aquellas mediadas por pasos automatizados. En las actividades con niveles de interacción más altos se ofrecen, en cambio, diversas opciones de acción, como la posibilidad de probar y controlar variables o desarrollar el razonamiento lógico y generar interacciones cognitivas, estéticas y afectivas, así como la inmersión y la participación simultánea de más de un visitante para fomentar el diálogo entre ellos (ROCHA, 2018).

En esta misma línea Rocha (2018) indica que investigadores como Allen (2004) y Oliveira et al. (2014) reclaman que la interacción física debe huir del "reduccionismo experimentalista", tener valor y objetivos educativos y llevar a interacciones de otros tipos, llamadas minds-on y hearts-on por Wagensberg (2001, 2005, 2006), las cuales serán desarrolladas en la sección dedicada al marco teórico.

Las investigaciones orientadas, justamente, a comprender mejor la experiencia de los visitantes han crecido en los últimos 30 años, con rápida expansión de los referentes teóricos y metodologías utilizadas (ROWE; BACHMAN-KISE, 2012). Y el crecimiento de estos estudios ha proporcionado nuevos entendimientos e información importante sobre las expectativas, preferencias e intereses de los mismos. Sin embargo, evaluar los resultados de las actividades ofrecidas en espacios de educación científica no formal continúa siendo un desafío (ROWE; O’BRIEN, 2016). Frente a un escenario regional tan fértil y con tantos aspectos por descubrir en lo referido a los estudios de público y, por otro lado, tan colmado de desafíos y debates en lo que corresponde a la interactividad, se vuelven indispensables estudios sobre cómo los visitantes interactúan con este tipo de propuestas expositivas.

En línea con ello, esta investigación trata de discutir cómo se da la interacción de adolescentes, un público poco frecuente en los museos de ciencias de la región, con una exhibición del CICyT Abremate ${ }^{4}$. También se busca renovar el conocimiento y los recursos de evaluación de procesos de aprendizaje en estos contextos, e indagar en la creación de sentidos por parte

\footnotetext{
${ }^{4}$ Integra las acciones de la red Cyted Musa Iberoamericana: Red de Museos y Centros de Ciencia y del Instituto Nacional de Comunicação Pública da Ciência e Tecnologia.
} 
de distintos públicos en actividades de educación no formal en espacios científico-culturales. Para ello se propone vincular dos ejes: por un lado, presentar datos concretos del registro de la interactividad en acción, es decir, sucediendo en un contexto específico, a partir de un estudio de caso y métodos cualitativos de indagación; por otro, continuar los esfuerzos orientados a revisar el concepto de interactividad y contrastarlo con los datos empíricos que disponemos.

\section{Marco teórico}

Dado que, tal como señala Allard (1998), la noción de aprendizaje es, en general, confusa, resulta indispensable especificar que en este trabajo nos referimos a actividades contextualizadas, que permiten internalizar herramientas culturales y cognitivas como parte de la participación en actividades socialmente significativas (ROWE; O’BRIEN, 2016).

El aprendizaje también remite aquí a procesos que favorecen el desarrollo de herramientas para crear, argumentar, discriminar fuentes confiables y realizar observaciones críticas. Tradicionalmente denominado en el ámbito de educación en ciencias como pensamiento de orden superior, este se asocia al conjunto de actividades mentales requeridas para el análisis de situaciones desconocidas, la resolución de problemas complejos y la toma de decisiones informadas (FURMAN et al., 2018). Metas que además se consideran fundamentales para la escolarización en el siglo XXI (ZOHAR, 2006).

Siguiendo la denominación previa, basada en la taxonomía clásica de Bloom (1956), las actividades de orden superior consisten en la adquisición, el ejercicio, la práctica de habilidades - no necesariamente de contenidos - para analizar, sintetizar y evaluar. Es decir, cualquier actividad cognitiva que supere la comprensión, memorización y recuperación de contenidos. Otros ejemplos de actividades cognitivas de orden superior son argumentar, hacer comparaciones, resolver problemas complejos, trabajar con controversias, identificar suposiciones subyacentes, formular preguntas de investigación, proponer hipótesis, planear experimentos y elaborar conclusiones (ZOHAR, 2006).

En contextos informales de aprendizaje (museos, exposiciones, zoológicos, acuarios o parques) se trata, además, de que suceda una actividad socialmente significativa que facilite, entre otros, el dominio de herramientas culturales para la comunicación y la cognición (ROWE; BACHMAN-KISE, 2012; WERTSCH, 1998). Y también de que se realice un proceso que siempre es activo, distribuido y situado social, cultural e históricamente (ROGOFF et al., 2003) y que, además, atañe tanto al desarrollo de la identidad como al dominio del conocimiento y habilidades prácticas (BELL et al., 2009).

Esta concepción del aprendizaje está alineada con las acepciones de interactividad que predominan en la literatura y en las propuestas - discursivas y materiales - de buena parte de los museos de ciencias en la actualidad. El concepto sería superador de la museografía tradicional y funcionaría como garantía para la generación de interés y procesos de aprendizaje en el sentido antes mencionado.

Wagensberg $(2001,2005,2006)$ es uno de los autores que desarrolla el concepto de interactividad y las expectativas asociadas. Este autor la define como sinónimo de conversación, en tanto diálogo en el que se ponen en juego, principalmente, las habilidades de pensamiento 
superior recién referidas, diferenciadas del contenido. Este mismo autor (WAGENSBERG, 2001), además, identifica tres modalidades de interactividad: Minds-on (mental): reflexionar o conversar con uno mismo, experimentar emociones sobre la inteligibilidad del mundo; Handson (manual): conversar con la naturaleza a través de la manipulación, recibir respuestas y provocaciones, generar preguntas y tomar decisiones, experimentar emociones provocadoras; Hearts-on (cultural): conversar entre los visitantes, experimentar emociones vinculadas a las identidades colectivas del entorno del museo.

La interactividad desde esta perspectiva aspira, entonces, a provocar y asegurar instancias de "conversación" del visitante con: la exhibición, él mismo y otros visitantes de tal forma que, en su máxima expresión, "el ciudadano viva las emociones del científico" y atraviese la experiencia de vincularse con el mundo - incluyendo fenómenos naturales, su propia mente y a otros seres humanos alrededor - desde "emociones inteligibles" (WAGENSBERG, 2001, p. 23). La interactividad así concebida es también una expectativa y una declaración de principios que, además, incluye un objetivo didáctico funcional al tipo aprendizaje antes desarrollado.

Sin embargo, en la práctica, las expectativas pueden concretarse o no. Y, en efecto, hay ejemplos que muestran que puede fallar. Oliveira et al. (2014) problematizan el concepto señalando casos en los que la "interactividad mental" - centro y objetivo máximo de la noción general de interactividad - se desarrolla en forma reducida, bien por falta de significatividad para el público, bien porque la "conversación" con los artefactos se detiene en el registro lúdico, sin llegar a desencadenar reflexiones y preguntas.

Allard (1998) también refiere un ejemplo que refleja la distancia que puede haber entre las expectativas y la práctica. En este caso se trata de un trabajo sobre un museo de paleontología en Canadá que puso en evidencia límites para alcanzar objetivos de aprendizaje cognitivo, a la vez que sorprendió con la realización de metas, aparentemente menos previstas en este caso, vinculadas al goce estético. Estos ejemplos son útiles para ilustrar la pertinencia de revisar las distancias y los puentes que existen entre expectativas y práctica en lo que respecta a interactividad.

Teniendo en cuenta las nociones de interactividad y aprendizaje referenciadas, avanzaremos en la comprensión de la experiencia museística interactiva tomando el estudio de caso mencionado de Abremate en Argentina.

\section{Metodología}

Se realizó un estudio exploratorio con métodos cualitativos para caracterizar las visitas de adolescentes a museos de ciencias. El registro audiovisual corresponde a las visitas realizadas por una muestra no representativa de grupos mixtos de adolescentes pertenecientes a escuelas públicas del Conurbano Bonaerense. Los visitantes completaron un cuestionario con sus datos de perfil, intereses y actitudes relacionados con la ciencia y la tecnología. La clasificación del material audiovisual se orientó a registrar la presencia, caracterizar y describir situaciones de interactividad y reacciones observables frente a las mismas, vinculadas a signos de aprendizaje. 


\section{El corpus}

Las visitas que conforman el corpus de este estudio fueron realizadas en el CICyT Abremate, cuyo objetivo es "poner la 'ciencia en acción', para tender puentes entre la comunidad educativa y para la promoción de la cultura científica a través de caminos de conocimiento interactivos que permitan el tránsito lúdico de los aprendizajes" (UNIVERSIDAD NACIONAL DE LANÚS, 2018). El principal mecanismo didáctico de la institución favorece la alfabetización científica es la interactividad: los visitantes pueden jugar con los módulos y superar la mera instancia contemplativa.

Abremate posee una exhibición permanente con 64 módulos interactivos que representan fenómenos de física, matemáticas, química y biología. Los dispositivos son de carácter electrónico y mecánico, se destacan por su tamaño y colores brillantes, pueden ser accionados de forma individual (presionar un comando), cooperativa (participación de varios visitantes) o competitiva (participación de equipos opuestos). Cada uno está acompañado por cartelería explicativa que refiere a la explicación del fenómeno científico o tecnológico e incluye un breve comentario sobre el modo de funcionamiento. Los módulos están en un espacio sectorizado por gigantografias colgantes que contextualizan históricamente los inventos científicos y tecnológicos representados (la Antigüedad, Los Grandes Imperios Antiguos, la Edad Media, el Renacimiento, la Edad Moderna y la Edad Contemporánea y Nuestro Tiempo).

En las visitas a Abremate para este estudio participaron cinco grupos de adolescentes (17 individuos en total) de entre 14 y 17 años, mixtos en cuanto a género, provenientes de escuelas secundarias públicas del Conurbano Bonaerense. Un visitante de cada grupo fue invitado a llevar consigo una cámara GoPro, que acompañó al grupo durante la visita (con duración aproximada de 20 minutos), para el registro de audio y vídeo desde la perspectiva del visitante. Las visitas se realizaron en forma libre. Los grupos visitaron el sector correspondiente a los períodos antiguo y Edad Media. En los cuestionarios aplicados, todos declararon interés por las materias escolares sobre ciencia y tecnología debido a que sienten curiosidad, llaman su atención o despiertan su interés. Las fuentes de información científica y tecnológica que utilizan habitualmente son: Internet; la escuela; los amigos; la televisión y la familia, en orden descendente según consumo.

\section{Codificación y clasificación de datos}

El material audiovisual fue codificado y clasificado con el software Dedoose 8.0.23, una aplicación que permite analizar datos cualitativos y mezclar métodos de investigación con fotos, audios y videos, entre otros. Dedoose se utilizó en este estudio para catalogar y agrupar, de acuerdo con criterios previamente establecidos, segmentos del registro audiovisual de acciones corporales, textuales y actitudinales de los visitantes que participaron en el mismo. La herramienta permitió la visualización y análisis exploratorio de la información registrada en las visitas por medio de técnicas cuali y cuantitativas. Complementaria y funcionalmente, se utilizó un protocolo de codificación desarrollado por la red de investigadores involucrados en el proyecto. Las dimensiones y categorías del protocolo fueron discutidas y revisadas durante el proceso de codificación, validadas por seis investigadoras-codificadoras, que también lo aplicaron en otros siete museos de ciencia de América Latina. 
El protocolo de codificación está dividido en dimensiones vinculadas a la noción de interactividad y a las expectativas asociadas. La funcionalidad de esta herramienta es detectar y caracterizar ocurrencias de expresiones de la interactividad entendida, en términos generales, como conversación, tal como fue definida en la sección 2 de este trabajo. Las dimensiones del protocolo incluyen: (1) sobre qué conversan los visitantes: el contenido de las conversaciones (experiencias previas, vivencias personales, temas ajenos al museo, temas científicos); (2) con qué o quién conversan/interactúan y cómo: cuál es el interlocutor (exhibición, mediador, otros visitantes) y cómo lo hacen (en forma activa o contemplativa); (3) qué efectos visibles generan las conversaciones/interactividades: provocación de cambios o emociones.

Las dimensiones y categorías fueron actualizadas durante el proceso de discusión y validación, lo cual dio como resultado una herramienta que combina aspectos teóricos y otros empíricos de la interactividad. Los últimos se inspiraron en las observaciones de los datos y complementaron el diseño teórico original de la herramienta. La Tabla 1 lista las dimensiones y categorías que integran esta herramienta.

\section{Estrategia de análisis}

En función del marco teórico presentado, el caso de estudio seleccionado y los datos recolectados, este trabajo plantea las siguientes preguntas para guiar el análisis: ¿Qué presencia tienen las escenas y situaciones de interactividad en las visitas realizadas? ¿En qué consisten las registrables y predominantes? ¿Hay una vinculación aprehensible entre la interactividad y el aprendizaje? (o, lo que es similar, ¿se realizan las expectativas en torno a la interactividad?). La estrategia de análisis consiste en poner en diálogo: (1) los datos filtrados a través de las dimensiones y categorías del protocolo antes referido; (2) la noción de interactividad, sus expectativas, aspiraciones y modalidades asociadas; y, (3) las preguntas de recién planteadas, en tanto guías de indagación.

\section{Resultados}

\section{Presencia de escenas y situaciones interactivas}

Dado que este trabajo es exploratorio y cualitativo, la cuantificación de resultados no es determinante para el análisis. De todas maneras, la contabilización de ocurrencias de las categorías que integran la herramienta de codificación permiten detectar y caracterizar, en forma aproximada, expresiones de la interactividad. En la Tabla 1 se contabilizan y detallan las ocurrencias ordenadas en forma descendente y por dimensión. 
Tabla 1 - Ocurrencias de categorías asociadas a la interactividad

\begin{tabular}{llc}
\hline \multicolumn{1}{c}{ Dimensiones } & \multicolumn{1}{c}{ Categorías } & Ocurrencias \\
\hline Contenido de conversaciones & \begin{tabular}{l}
\multicolumn{1}{c}{ Sobre la exhibición (funcionamiento, diseño, } \\
experiencia museal)
\end{tabular} & 37 \\
& Temas de ciencias & 14 \\
& Asociación con experiencias anteriores y vivencias & 11 \\
& personales & \\
& Temas no relacionados con el contenido de la & 4 \\
& exhibición & \\
& Percepciones sobre determinado tema científico & 2 \\
& Percepciones sobre científicos & 1 \\
Contrapartes y formas de & Visitante-exhibición + actividad interactiva & 65 \\
conversación/interactividad & Visitante-visitante & 51 \\
& Visitante-exhibición + lectura de panel & 14 \\
& Visitante-exhibición + interacción contemplativa & 9 \\
Efectos de conversación & Visitante-mediador & 1 \\
interactividad & Cambios de ideas (acuerdo/desacuerdo) & 3 \\
Total de ocurrencias & Expresión de emociones & 18 \\
\hline
\end{tabular}

Fuente: elaboración de los autores.

Entendida, entonces, la interactividad como sinónimo de "conversación", en tanto diálogo con la exhibición, con uno mismo y con otros visitantes en el que se ponen en juego, principalmente, habilidades - no solo contenido - en el plano de la "emoción inteligible" (WAGENSBERG, 2001, p. 23); y dado el instrumento de codificación de datos alineado con dicha concepción de la interactividad, lo que indica este primer conteo es que hay escenas y situaciones de interactividad que se realizan en las visitas que forman parte del caso de estudio Abremate.

Además, este conteo también indica que algunas expresiones de la interactividad predominan por sobre otras. Por ejemplo, las tres principales ocurrencias indican que: 65 de ellas refieren a situaciones en las que los visitantes interactúan con la exhibición en forma activa; 51 corresponden a escenas en las que el grupo de visitantes interactúa entre sí; y 37 refieren a episodios en los que los visitantes conversan sobre la exhibición, su funcionamiento, diseño y la experiencia en general.

Respecto a la primera pregunta planteada en este trabajo, acerca de la presencia de escenas y situaciones de interactividad, podemos observar que están, se pueden identificar y capturar; así como que los episodios en las que más se expresa son aquellos en los que: los visitantes interactúan activamente con la exhibición; entre sí y conversan sobre cómo funciona la exhibición o cómo desenvolverse para interactuar con ella.

Aunque en forma más tenue, hay otras expresiones de interactividad que también tienen lugar en estas visitas. Por ejemplo: las emociones emergen 18 veces; los temas de ciencias surgen en 17 ocasiones durante el total de recorridos; las lecturas de paneles 14 veces y las conversaciones sobre experiencias previas y vivencias personales presentan 11 ocurrencias. 
Luego, la interactividad de los visitantes con la exhibición en forma contemplativa se detecta en 9 ocasiones; las conversaciones en torno a temas ajenos a la exhibición 4 veces; efectos de la interactividad expresados en cambios explícitos de ideas presenta 3 ocurrencias; percepciones sobre temas científicos se expresan apenas 2 veces y percepciones sobre científicos (la profesión) e interacciones con mediadores 1 sola vez.

En el apartado que sigue intentaremos ingresar en niveles más finos buscando reconocer parcialmente cómo y de qué manera se expresa esta interactividad. En la forma que adquiere aquí la interactividad, las categorías o expresiones menos presentes y ausentes podrían estar condicionando qué, cómo y cuánto emergen los aspectos cualitativos que se expresan con más contundencia.

\section{En qué consiste la interactividad, de qué se trata}

Tal como señala Wagensberg (2001, p. 23) respecto a la interactividad manual o emoción provocadora, "pulsar un botón para poner en marcha un proceso preprogramado es solo una caricatura" que permanece lejos de la expectativa de la interactividad vinculada a que el visitante "se introduzca en la piel del científico".

Son varios los autores que encienden las señales de alerta en este sentido, remarcan la necesidad - indispensable - de superar el reduccionismo experimentalista e invitan a discriminar entre la provocación de reacciones mecánicas y los procesos de aprendizaje antes referidos, aquellos orientados abrir caminos y crear herramientas para vincularse, comprender y curiosear sobre el mundo con recursos de la ciencia (ALLEN, 2004; OLIVEIRA et al., 2014; ROCHA, 2018). En este mismo sentido, en referencia a las actividades de experimentación sobre objetos y procesos naturales en el ámbito formal de enseñanza de las ciencias, Furman et al. (2018, p. 95) diferencian entre presentar "protocolos predefinidos con pasos a seguir para la comprobación de fenómenos" y generar condiciones para la formulación de hipótesis, el diseño experimental, discutir parte del procedimiento involucrado, hacer predicciones, ponerlas a prueba y evaluar datos para elaborar conclusiones.

Teniendo en cuenta estas advertencias, cabe preguntar de qué se trata la interactividad en nuestro caso de estudio. La combinación de las tres principales ocurrencias mencionadas en el apartado previo puede brindar algo de información al respecto.

La principal expresión de la interactividad en estas visitas corresponde a situaciones en las que, simultáneamente, los visitantes interactúan/conversan con la exhibición (ej. levantan pesas; giran una manivela; caminan dentro de una rueda u observan a través de lupas), a la vez que conversan con otros visitantes de su grupo y comentan cómo se desenvuelven en la exhibición o cómo hacer para lograrlo. A seguir, ejemplos de las mencionadas conversaciones sobre la exhibición ${ }^{5}$, su funcionamiento y diseño; y la Figura 1 muestra la combinación frecuente de situaciones que caracteriza las visitas.

\footnotetext{
${ }^{5}$ Nota: V refiere a visitantes y el número contiguo los discrimina entre sí. Mód. refiere a módulo expositivo.
} 
1) V1: yo necesito ayuda acá. V2: ¿qué biciste? Lo rompiste. V1: Ay, porque lo intenté poner y se me bajó. [Mód. Poleas y aparejos].

2) V1:zporquésuenaesto?V2: haceelruidodelcoso. [Mód.PrincipiodeArquímedes].

3) V1: No me preguntes para qué sirve porque ni idea. V2: Se mueve eso. V1: No se movió. V2: Sí se movió, fijate, dalo vuelta. V1: Ah, sí se movió. V2: Ah sí, se movió. [Mód. Teorema de Pitágoras].

Figura 1 - Interactividad/conversaciones de los visitantes

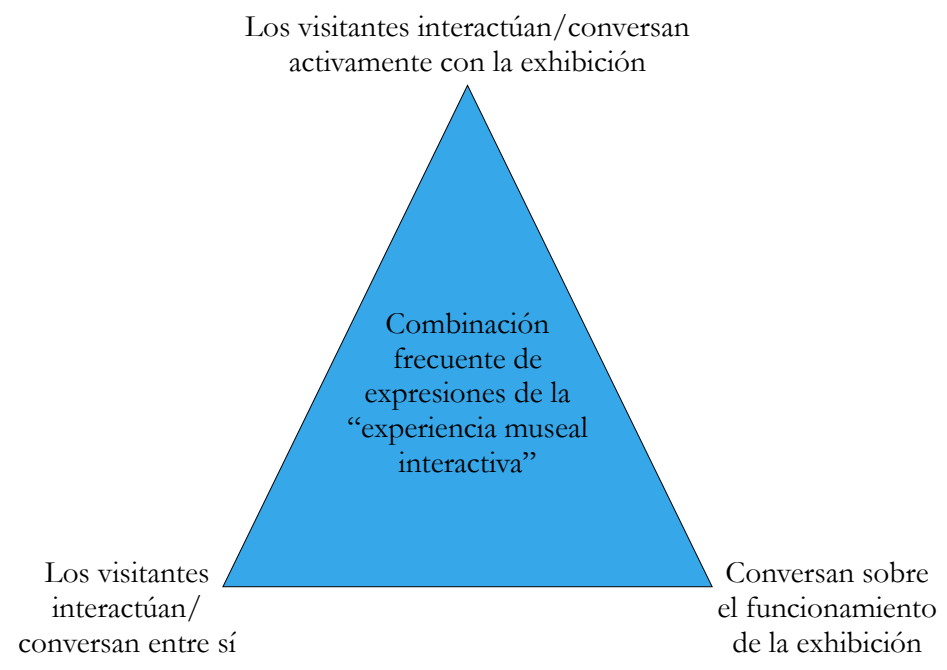

Fuente: elaboración de los autores.

Las situaciones en las que los visitantes interactúan activamente con la exhibición y entre sí, en simultáneo, es la que más se repite ( 35 veces). Por otro lado, los visitantes interactúan con la exhibición a la vez que conversan sobre el funcionamiento de la misma en 24 ocasiones; y conversan entre sí sobre esos mismos temas 23 veces.

Es significativo que entre las categorías originalmente diseñadas en este proyecto, no estuvo la referida a diálogos, comentarios y preguntas sobre la exhibición, su funcionamiento, diseño y la experiencia museal, es decir, la más frecuente entre los contenidos de las conversaciones. De hecho, fue creada a partir de las repeticiones - imprevistas desde el diseño original de la herramienta de codificación - registradas en los propios datos (parte de la construcción teórica y empírica de la herramienta explicitada en la sección Codificación y clasificación de datos).

Esto podría vincularse con que las conversaciones sobre cómo interactuar manualmente con la exhibición (cómo hacer funcionar un módulo; para qué lado hacer girar una rueda y comentarios similares sobre el quehacer mismo de la manipulación de los artefactos) escapan a las expectativas de la interactividad, en tanto no llegan a reflejar, tal como sostienen Oliveira et al. (2014, p. 4), "elementos de interacción que estimulan el funcionamiento de la 
mente, instigando a los visitantes a emprender un 'ejercicio' mental, elaborando cuestiones, solucionando problemas, creando analogías y percibiendo contradicciones".

En este sentido, si la aspiración de la interactividad es, aunque sea parcialmente, la emoción inteligible, la conversación con la naturaleza, provocar para generar preguntas y tomar decisiones vinculadas a fenómenos naturales, entre otros, las expresiones antes referidas no llegan a realizar el proceso de interactividad en sentido acabado. Es decir, los signos aprehensibles del proceso muestran que el mismo se realiza, pero no en su máxima expresión.

De todas maneras, el camino está iniciado. Y, en ocasiones, las conversaciones sobre la exhibición no concluyen en los mecanismos de manipulación y alcanzan a rozar temas de ciencia e, incluso, esbozan habilidades, razonamientos cercanos / similares a la inteligibilidad de la ciencia. En Abremate hay módulos que provocan - en forma repetida - expresiones más cercanas a las expectativas de la interactividad. Siguen las conversaciones generadas en torno al módulo Poleas y aparejos.

4) V1: este al tener una sola polea, pesa más. V2: todas las bolsas pesan todas lo mismo, igual. V1: este al tener dos poleas pesa menos. V3: $Y$ esta al tener 4 poleas la puedo levantar. V2: yo quiero intentar este. 5) V1: Mirá, este es así. Este es más pesado porque solo tiene 1, ese es menos pesado porque tiene 2 y ese como tiene 4, bueno, es bien liviano. ¿Listo? V2: este creo que es el menos pesado, ah, no, este. V3: porque cada polea divide el peso. V2: este tiene 2 poleas, a ver este... V1: Mirá, mirá esto, Joaquin, mirá mi fuerza. V2: oooohbh dios mío. V3: yo también estoy haciendo fuerza, ¿ves? V1: levantalo, levantalo, levantalo rápido. V2: abi tenés, lo dejé caer rápido. Acá se supone que este...ंlo suelto? V1: sí. V2: ah, muy bien, lo solté y no pasó nada. 6) V1: ¿qué habiamos estudiado de esto en la escuela? ¿Vos estudiaste esto ya en las escuelas? ¿Acerca de las poleas, en física? V2: No, pero sé que cuantas más poleas tiene, más fácil es moverlo. V1: si, las poleas lo que hacen es dividir por dos el peso, es decir, dividen por dos la fuerza que se hace hacia abajo. ¿Me entendés más o menos, no? Entonces cuando tenés 2 poleas, ahí se está dividiendo por 4 y allá tiene 4 poleas, se divide $2 \times 2 \times 2 \times 2$ o sea, $2 \times 2=4$, $\mathrm{x} 2=8$, $\mathrm{x} 2=16$. ¡Una locura!

Si, como sostiene Allen (2004), la meta de los museos de ciencias es poner al visitante en un rol de aprendiz activo en el que pueda experimentar, generar hipótesis, interpretar y delinear conclusiones y, de acuerdo con Wagensberg (2001), también se trata de "conversar" con los fenómenos naturales, con otros y dialogar con el mundo desde la "emoción inteligible", los últimos ejemplos parecen avecinarse más a tales expectativas: el fragmento 4 refleja interpretaciones, curiosidad e interés; el 5 experimentaciones e interpretaciones; y el 6 referencias a conocimientos previos y explicaciones, entre otras actividades que se acercan a las aspiraciones de la interactividad antes mencionadas.

Estos fragmentos también se acercan un poco más a las expectativas, en tanto integran el grupo de conversaciones cuyo contenido refiere a temas de ciencia (los cuales, en total, presentan 17 ocurrencias, ver tabla 1). A continuación se citan algunos ejemplos más de fragmentos de conversaciones codificados dentro de esta categoría. 
7) V1: ¿y eso qué onda Nico? ¿Qué es? ¿Qué hace? V2: No sé, leé. V1: [señalando cartelería del módulo] ah, yo habia leído muchopero mucho sobre este tipo Arquímedes cuando era chiquito. Era un libro de experimentos con agua y este [en referencia al módulo] era uno de lo más importantes que habia descubierto Arquímedes. V2: ¿y qué decía? V1: lo que decía es que el rey necesitaba saber cuánto era la masa de su corona porque no sabia si era de oro, completamente de oro, o si solamente estaba bañado en oro. Entonces la metió dentro de la pileta, como está acá y se dio cuenta que cuanto más peso tenía era porque tenía más oro. No me acuerdo cómo era la historia completa, lo leí cuando tenía 7 años así que no tengo ni idea abora. V2: así que podrías estar mintiendo. V1: podría estar mintiendo, sí, tranquilamente. [Mód. Principio de Arquímedes]. 8) V1: igual, este es más chiquito, por eso no hay...este es más chiquito y este es más grande. No son igual proporción [en referencia a los cuadrados del módulo]. V2: no.. V3: no, se supone que no. V2: estos dos forman este. V2: se supone que este, más la suma de este, te da esto [en referencia a los cuadrados]. Si fueran de igual proporción esto tendría que ser el doble. Yo creo que la fórmula era... V1: Pi por raíz al cuadrado. V2: $A$ la $2+B$ al cuadrado es $=a C$ al cuadrado. V1: Aguante matemáticas. [Mód. Teorema de Pitágoras].

En los fragmentos 4 a 8 se identifican explicaciones, referencias a conocimientos previos e hipótesis. El contenido de estas conversaciones se extiende algo más allá de los comentarios e intentos por desentrañar la acción mecánica y, en efecto, rozan más de cerca "la experimentación, la generación de hipótesis, interpretaciones y conclusiones" que, como destaca Allen (2004, p. 4, traducción nuestra), son eje y aspiraciones centrales de los museos de ciencias.

Sin embargo, como detallamos en la sección Presencia de escenas y situaciones interactivas, la presencia de esta categoría (temas de ciencia), no solo es menor comparada con las predominantes (mientras que las conversaciones sobre la exhibición, su funcionamiento y diseño se identifican 37 veces, las que incorporan temas de ciencia se registran en 17 ocasiones), sino que, además, hay oportunidades en las que ese contenido (temas de ciencia) se interrumpe o diluye. Esto resulta central en la descripción y comprensión de la experiencia porque, tal como mencionamos en el marco teórico, la conversación que recupera, recuerda, reproduce y aplica temas de ciencia, si bien puede constituir un primer paso hacia la interactividad y el tipo de aprendizaje referido (ALLARD, 1998), no alcanza a constituir el tipo de actividad que "enseña a pensar" (ZOHAR, 2006, p. 158). En tal caso tiene valor como escalón inicial hacia el desarrollo de habilidades para analizar, sintetizar y evaluar, entre otras (ZOHAR, 2006). Recuperamos a continuación algunos fragmentos de situaciones interactivas y conversaciones incipientes sobre temas de ciencias que se diluyen.

9) V3: Nooo [quitándole una cuerda a su compañero]. V2: No, después lo saco es para colgarme de esa [disputando la cuerda con su compañera e intentando colgarla del módulo]. V1: tremendas discusiones entre ustedes dos. Justo tocaron los más quilomberos, je. 'Multiplicá tu fuerza' se llama este. Ob wow, jvas a revolar vos! Vas a volar más que Superman. V2: soltá, soltá [continúa la disputa por la cuerda], ¿quieren verme volar chicos? V3: ¡No! [la discusión 
entre V3 y V2 continúa]. V1: a ver si pongo esto así. V2: Toto, mirá esto. V1: ¿qué querés hacer? ¡Wow! Casi me golpeo. ¡Bien! A ver, esperá, agarrá y poné este [en relación a las cuerdas del módulo]. V2: ¿listo? V1: Sí. No, a ver esperá, mantenelo, mantenelo [colocando otra cuerda en otra parte del módulo]. Y abora hacemos así, con más peso requerido. Estamos multiplicando la fuerza, por lo menos eso dice el título de la actividad. V2: Bueno, listo [mientras se vuelve a colgar de otra cuerda]. V1: Yo creo que sí. ¿Por qué hacés así? iJulieta! [señalando a V2]. [Continúa una discusión entre V2 y V3 sobre cómo utilizar el módulo, en torno a si se trata de tocar o destruir]. V3: jse llama probibido no tocar, no destruir! V1: A ver, rompé el lugar como dice Julieta [en referencia a V2 que continúa colgándose de las cuerdas]. Esto sí que es el casting de Tarzan, abahaha [simulando el grito de Tarzán]. [Mód. La Palanca]. 10) V1:jGo Mati, go! ¿Nos podrías explicar qué está pasando acá? V2: yo, justamente, no. V1: Bueno, Nico... V3: hay un chico que se quiere romper la cabeza, eso pasa. V1: Nico, ¿sabés lo que pasa acá? V2: subi vos Toto con la cámara. V1: sí sí, subo yo, quiero. V3: dale, dale quiero que pagues la cámara. V2: dale, subite, si nosotros te tenemos por cualquier cosa, en serio [con ironía]. ¡Dale! ¡Dale! V1: te estoy observando, eh, acordate. jTengo un miedo! V2: te tenés que agarrar de... [varios traspiés con el módulo, todos se ríen]. V1: es curioso cómo funciona... ¿Y esto dónde lo usaban? Me acuerdo de algún lado... V2: en Utilisima [corresponde un programa argentino de TV, muy tradicional, sobre cocina]. V1: ab cierto [...] para moler los granos de maí: V2: ipero en dónde, no para qué! V1: [lectura de panel] en la Mesopotamia [el grupo no permanece al lado de V1 que lee el panel y continúa el recorrido hacia otro módulo]. [Mód. La Noria].

El fragmento 9 refleja una situación en la que, a pesar de estar presentes y confluir condiciones favorables para la interactividad (manipulación; inicio de búsqueda de explicación "Y ahora hacemos así, con más peso requerido. Estamos multiplicando la fuerza, por lo menos eso dice el título de la actividad"; interés; curiosidad; referencia a conocimientos previos e interacción entre pares, entre otras), la experiencia se diluye en una discusión entre miembros del grupo en torno al modo de interacción con la exhibición y a cómo debería hacerse, si tocándola o destruyéndola. El fragmento 10 también evidencia una situación en la que hay expresiones de interés, curiosidad y referencias a temas de la vida cotidiana, así como diversos intentos orientados a avanzar en la indagación del artefacto y, sin embargo, la dinámica del grupo - que podría potenciar la experiencia interactiva - diluye el proceso de descubrimiento del artefacto.

La lectura de paneles es otro componente que puede generar y acompañar la interactividad, provocándola o profundizándola. Como se vio en la Tabla 1, esta expresión de la interactividad, aunque está entre las más tenues, está presente en el caso de estudio. El mismo fragmento 10 también ejemplifica una situación en la que la lectura del panel, aunque podría enriquecer y contener la experiencia interactiva, se interrumpe. Y los fragmentos de lecturas de panel que se interrumpen o diluyen también reflejan este límite.

11) V1: para mi también eran del Egipto [...] los egipcios eran unos genios, literal. V2: abi dice [en referencia al panel, mientras lo mira y lee en voz 
baja] V1: creo que Peraccio dijo unos $100 \mathrm{~kg}$. Igual no me suena, si no estaríamos levantando $50 \mathrm{~kg}$. con una sola mano... V4: no sé, ni idea... [el grupo se dispersa y continúa el recorrido]. [Mód. Poleas y Aparejos]. 12) V1: ¿y esto qué explica, 'chicos físicos?' [mientras observan el módulo y uno de los visitantes jala de una cuerda]. V2: abi me mataste. ¿No es esto? Enfocá la explicación para que la gente lo pueda ver [en referencia al panel] V1: [lee el panel] Elprincipio de la palanca. La palanca fue uno de los primeros inventos, pequeños e inmenso [...]. Fue una máquina simple de creador desconocido al igual que la rueda, surgió hace unos miles de años y sin ella no bubiese sido posible levantar los grandes monumentos de piedras europeos [el grupo se dispersa y V1 queda solo leyendo el panel]. ¿Y en qué se usa esto actualmente, alguien lo sabe? [pregunta al grupo y le responden con alguna broma] ¿Nadie lo sabe? En las grúas se usa esto. V2: Ah sí, qué inteligente [con ironía]. [Mód. La Palanca].

Las asociaciones con experiencias previas o vivencias personales también forman parte de las expectativas explícitas de la interactividad. Como sostiene Wagensberg (2001, p. 23), esta implica "alejarse de un experimento del museo asociando ideas con la vida cotidiana, con otros casos que puedan responder a la misma esencia".

En este estudio se registran 11 situaciones en las que las conversaciones incluyen asociaciones con experiencias previas o vivencias. Nuevamente, la presencia de este tópico es mucho menor si se lo compara, por ejemplo, con las conversaciones predominantes sobre el funcionamiento de la exhibición. Pero, además, en términos cualitativos, de manera similar a lo que sucede con la lectura de paneles, se trata de contenidos que, en lugar de desarrollarse, de funcionar como un trampolín para saltar hacia las habilidades, se interrumpen. No llegan a integrar una situación en la que, por ejemplo, se establezcan relaciones con la vida cotidiana y a partir de allí surjan inferencias (FURMAN et al., 2018), o se construyan nuevas ideas a partir de ellas. Siguen asociaciones con experiencias personales y previas que se interrumpen.

13) V1: a ver si puedo ver la mano. V2: grandoootas. V3: jajajaja V1: se ve una calidad mucho mejor que las cámaras nuevas que están sacando full hd $4 k$. V1: holaaaa. V2: hacé caras estúpidas. V1 [hace un gesto con la mano]. V2: ¿por qué haces así? Está en la cámara. V1: ¿Se ve bien? V2: sí, está en la cámara. V1: [cara de sorpresa] holaaaa. [Mód. Lupas de agua]. 14) V1: ¿y eso qué onda Nico? ¿Qué es? ¿Qué hace? V2: No sé, leé. V1: [señalando cartelería del módulo] ah, yo había leído mucho pero mucho sobre este tipo Arquímedes cuando era chiquito. Era un libro de experimentos con agua y este era uno de lo más importantes que babía descubierto Arquimedes. V2: ¿y qué decía? V1: lo que decía es que el rey necesitaba saber cuánto era la masa de su corona porque no sabía si era de oro, completamente de oro o si solamente estaba bañado en oro. Entonces la metió dentro de la pileta, como está acá y se dio cuenta que cuanto más peso tenía era porque tenía más oro. No me acuerdo cómo era la historia completa, lo leí cuando tenía 7 años asi que no tengo ni idea abora. V2: asi que podrías estar mintiendo. V1: podría estar mintiendo sí tranquilamente. [Mód. Principio de Arquímedes]. 
A partir de los ejemplos repasados en esta sección podemos concluir que, en el caso de las cinco visitas realizadas a Abremate, la interactividad está presente y hay condiciones dadas para profundizar la experiencia en el sentido de las expectativas de la interactividad y la concepción de aprendizaje que desarrollamos.

\section{Vínculos entre interactividad y aprendizaje}

En respuesta a las preguntas que guiaron nuestro análisis pudimos registrar, por un lado, que la interactividad está presente porque hay conversación. Por otro lado, observamos que la forma en que ésta se expresa es, principal aunque no exclusivamente, en situaciones que combinan interacciones con la exhibición, entre los visitantes y en las que predomina como tema de conversación desentrañar la manipulación o comentar acciones relacionadas con la misma.

También vimos que, aunque en menor magnitud, hay módulos (como Poleas y aparejos) que desencadenan ejercicios mentales algo más cercanos a las expectativas de la interactividad (planteo de hipótesis, asociaciones con conocimientos previos, desarrollo de interpretaciones y conclusiones). Sin embargo, si recuperamos la noción de interactividad y sus expectativas intrínsecas, es posible reconocer que el proceso puede - y quiere - ser una experiencia más profunda, los ejercicios más agudos y los puentes entre los estímulos de la exhibición y la "emoción sobre la inteligibilidad del mundo" de la que habla Wagensberg (2001, p. 23) más sólidos y claros.

El recorrido teórico y empírico que hemos hecho permite interpretar que la experiencia interactiva y los lazos con el aprendizaje ya comenzaron, existen y las condiciones están dadas: la manipulación es un hecho; la curiosidad aparece; las referencias a información previa y experiencias cotidianas se citan espontáneamente; la explicación y el interés en ocasiones se expresan. Pero la observación de los datos también refleja que aún hay mucho camino por recorrer, por ejemplo, provocando situaciones en las que la experimentación sobre objetos y procesos crezca con la discusión de un procedimiento; la posibilidad de proponer un diseño experimental del mismo; la formulación de hipótesis y predicciones; la puesta a prueba y evaluación de datos y conclusiones; la relación con la vida cotidiana ligada a inferencias; la síntesis y relación de conceptos; la reflexión sobre errores y dificultades y el análisis de situaciones desconocidas, entre otros (FURMAN et al., 2018).

La interacción del visitante con el objeto y el discurso expositivo tiene potencial de promover procesos cognitivos y desarrollar habilidades relacionadas con el aprendizaje, la investigación científica y el análisis crítico. Algunas de estas habilidades son la identificación, observación, nombramiento, afirmación, comparación, caracterización, estrategia, suposición, análisis, evaluación, conclusión y generalización. La exposición también puede posibilitar relación dialógica entre los diversos actores involucrados, por ejemplo, con mediadores de la institución, enriqueciendo la construcción colectiva del conocimiento (ROCHA, 2018).

Llegados a este punto podemos responder parcialmente la última - y más compleja - pregunta planteada como guía para nuestro análisis sosteniendo que, dados los datos disponibles en este caso, hay un camino emprendido hacia la interacción y el aprendizaje. Y también que ambos procesos podrían profundizarse generando más oportunidades para experimentar "actividades que enseñan a pensar" y ejerciten "emociones sobre la inteligibilidad del mundo" (WAGENSBERG, 2001, p. 23). 


\section{Reflexiones finales}

Estar en camino no es poco. Es posible ajustar las propuestas a través de la creatividad, la emoción y la mediación como recursos. También es clave revisar continuamente la noción de interactividad, recortar objetivos accesibles pero cercanos a las expectativas y poner a prueba mecanismos que lo validen, es decir, aplicar "el método de la emoción inteligible" (WAGENSBERG, 2001, p. 23).

Tal como señala Wagensberg (2001), el mejor método para imaginar, diseñar y producir exhibiciones científicas es el propio método científico. A lo cual agregamos que también resulta necesario actualizar el paradigma, por ejemplo, incorporando la experiencia de los visitantes desde su propia perspectiva.

En línea con ello, investigaciones futuras que tomen en consideración este trabajo, y el proyecto general en el que se incerta, tendrán la oportunidad de perfeccionar sus enfoques y resultados incorporando los aprendizajes de nuestra experiencia, en particular, aquellos elementos que atienden la perspectiva de los visitantes. Como se mencionó previamente, el protocolo original de indagación utilizado en este estudio se construyó de acuerdo con las expectativas máximas de la interactividad y el aprendizaje. Esto obligó, en la etapa de ordenamiento de los datos y su análisis, a reformular los códigos y categorías incorporando datos de la realidad que, precisamente, se alejan de las expectativas, por ejemplo, los diálogos, comentarios y preguntas sobre la exhibición, su funcionamiento y diseño que, finalmente, representaron los contenidos más frecuentes entre las conversaciones recolectadas. Este ajuste metodológico, lejos de representar una dificultad, constituyó un hallazgo metodológico e informativo sobre los datos. Y puso en primer plano los enlaces, la bisagra, la articulación entre expectativas y hechos empíricos vinculados a la interactividad. El dato amerita ser recuperado en trabajos futuros como guía de indagación, para continuar avanzando en la comprensión de los giros y oscilaciones que caracterizan el pivote entre la idea y la realización de la interactividad.

También puede resultar interesante cuestionar el orden establecido, por ejemplo, la hegemonía de la interactividad, y combinarla con otras estrategias, bien para enriquecerla, bien para evaluar y comparar. Si bien, como señala Allen (2004), la interactividad física tiene motivos para ser hegemónica porque es el mecanismo que permite acceder a fenómenos clave del mundo natural, promueve el compromiso, la comprensión y el recuerdo de las exposiciones, también es cierto que más no necesariamente es mejor. Esto sugiere que, si bien reconocemos el poder de las experiencias interactivas, podemos desconfiar, al menos, de las propuestas reduccionistas y reconocer que estas pueden no ser suficientes a la hora de generar experiencias más poderosas, memorables y atractivas en los espacios de educación no formal.

Tal como sostiene Alderoqui (2017, p. 109), "esta perspectiva reclama instalar situaciones de conversación verdadera”. En línea con ello, consideramos que en nuestro caso de estudio hay niveles y tipos de conversación que por ahora están vacantes y que se pueden poner a prueba para generar puentes creativos entre los contenidos y las habilidades para pensar (ZOHAR, 2006), es decir, para renovar y ajustar el pivote entre las expectativas y los hechos empíricos en las experiencias museales interactivas. 


\section{Agradecimientos}

Los autores agradecen el apoyo del Conselho Nacional de Desenvolvimento Científico e Tecnológico (CNPq), Casa de Oswaldo Cruz (FIOCRUZ) y Fundação Carlos Chagas Filho de Amparo à Pesquisa do Estado do Rio de Janeiro (Faperj).

\section{Referencias}

ALDEROQUI, S. Elogio de los visitantes. In: BIALOGORSKI, M.; RECA, M. M. (comp.). Museos y visitantes: ensayos sobre estudios de público en Argentina. Buenos Aires: ICOM Argentina, 2017. p. 95-121.

ALLARD, M. et al. Guide de planification et d'évaluation dês programmes éducatifs. Montreal: éditions Logiques, 1998.

ALLEN, S. Designs for learning: studying science museums exhibits that do more than entertain. Science Education, Hoboken, v. 88, n. S1, p. S17-S33, 2004. DOI: https://doi. org/10.1002/sce.20016.

ASENSIO, M. Prólogo. In: BIALOGORSKI, M.; RECA, M. (comp.). Museos y visitantes: ensayos sobre estudios de público en Argentina. Buenos Aires: ICOM Argentina, 2017. p. 7-17.

BELL, P. et al. (ed.). Learning science in informal environments: people, places, and pursuits: a report of the National Research Council of the National Academies. Washington, DC: The National Academies Press, 2009.

BLOOM, B. S. Taxonomy of educational objectives, handbook 1: cognitive domain. New York: Longmans, 1956.

CASTILLA, A. La profesionalización de los museos en Argentina: desde la centralidad de los objetos a la prioridad del visitante. Caiana: revista de historia del arte y cultura visual, Buenos Aires, n. 10, p. 137-45, 2017. Recuperado de: http:/ / caiana.caia.org.ar/resources/ uploads/10-pdf/castilla_pdf.pdf. Visitado en: 2 abr. 2019.

CAZELLI, S.; COIMBRA, C. A. Q. Avaliação formal na educação não formal. Rio de Janeiro: Associação Brasileira de Avaliação Educacional, 2008.

CAZELLI, S.; MARANDINO, M.; STUDART; D. C. Educação e comunicação em museus de ciência: aspectos históricos, pesquisa e prática. In: GOUVEA, G.; MARANDINO, M.; LEAL, M. C. (org.). Educação e museu: a construção social do caráter educativo dos museus de ciência. Rio de Janeiro: Access, 2003. p. 83-106.

FURMAN, M. et al. Abriendo la "caja negra" del aula de ciencias: un estudio sobre la relación entre las prácticas de enseñanza sobre el cuerpo humano y las capacidades de pensamiento que se promueven en los alumnos de séptimo grado. Enseñanza de las Ciencias, Barcelona, v. 36, n. 2, p. 81-103, 2018.

MARANDINO, M. Faz sentido ainda propor a separação entre os termos educação formal, não formal e informal? Ciência \& Educação, Bauru, v. 23, n. 4, p. 811-816, 2017. DOI: https://doi.org/10.1590/1516-731320170030001. 
MASSARANI, L. et al. RedPOP: 25 años de red en comunicación de la ciencia en América Latina. Journal of Science Communication, Trieste, v. 14, n. 3, 2015.

OLIVEIRA, B. J. et al. O fetiche da interatividade em dispositivos museais: eficácia ou frustração na difusão do conhecimento científico. Museologia e Patrimônio, Rio de Janeiro, v. 7, n. 1, p. 21-32, 2014.

PEDERSOLI, C. Los museos interactivos de ciencias como mediadores en la construcción del conocimiento. 2003. Disertación (Licenciatura en Ciencias de la Educación) - Facultad de Humanidades y Ciencias de la Educación, Universidad Nacional de La Plata, La Plata, Argentina, 2003.

ROCHA, J. N. Museus e centros de ciências itinerantes: análise das exposições na perspectiva da alfabetização científica. 2018. Tese (Doutorado em Educação) - Faculdade de Educação, Universidade de São Paulo, São Paulo, 2018.

ROGOFF, B. et al. Firsthand learning through intent participation. Annual Review of Psychology, Palo Alto, v. 54, n. 1, p. 175-203, 2003. DOI: https://doi.org/10.1146/annurev. psych.54.101601.145118.

ROWE, S.; BACHMAN-KISE, J. Mediated action as a framework for exploring learning in informal settings. In: ASH, D.; RAHM, J.; MELBER, L. (ed.). Putting theory into practice. Rotterdam: Sense, 2012. p. 143-163.

ROWE, S.; O’BRIEN, S. Pesquisa sobre aprendizagem em museus: um campo em busca de foco? In: DIVULGAÇÃO científica e museus de ciências: o olhar do visitante: memórias do evento. Rio de Janeiro: Museu da Vida, 2016. p. 9-22.

\section{UNIVERSIDAD NACIONAL DE LANÚS. Centro Interactivo de Ciencia y}

Tecnología: CICy'T abremate: fundamentación pedagógica. Recuperado de: http://www. unla.edu.ar/index.php/abremate-fundamentacion-pedagogica. Visitado en: 8 jul. 2018.

WAGENSBERG, J. Principios fundamentales de la museología científica moderna. B.MM, Barcelona, n. 55, p. 22-24, abr./jun. 2001.

WAGENSBERG, J. The "total” museum, a tool for social change. História, Ciências, Saúde - Manguinhos, Rio de Janeiro, v. 12, supl. 0, p. 309-321, 2005. DOI: http://dx.doi. org/10.1590/S0104-59702005000400015.

WAGENSBERG, J. et al. Cosmocaixa: el museo total por conversación entre arquitectos y museólogos. [Madrid]: Sacyr, 2006.

WERTSCH, J. V. Mind as action. New York: Oxford University Press, 1998.

ZOHAR, A. El pensamiento de orden superior en las clases de ciencias: objetivos, medios y resultados de investigación. Enseñanza de Las Ciencias, Barcelona, v. 24, n. 2, p. 157-172, 2006.

Artigo recebido em 13/07/2018. Aceito em 28/11/2018.

Contacto: Programa de Pós-graduação em Divulgação da Ciência, Tecnologia e Saúde, Casa de Oswaldo Cruz, Fundação Oswaldo Cruz, Av. Brasil, 4365, Manguinhos, Rio de Janeiro, RJ, 21040-360, Brasil. 